\title{
Beam Scanning UHF RFID Reader Antenna with High Gain and Wide Axial Ratio Beamwidth
}

\begin{abstract}
A novel ultra-high-frequency (UHF) RFID reader antenna with 2 dimensional (2D) beam-scanning capability and a wide $3 \mathrm{~dB}$ axial ratio (AR) beamwidth of over $136^{\circ}$ is presented. The proposed antenna consists of three concentric metal rings, each having a branch wire to stimulate circular polarization (CP) and a separate feed. The antenna is able to perform a 2D beam-scan with a relatively stable gain $(9-11 \mathrm{dBic})$ from $0^{\circ}$ to $360^{\circ}$ in azimuth, and $-40^{\circ}$ to $40^{\circ}$ in zenith. The minima of the antenna's AR beamwidth follows the peak gain direction, giving an equivalent $3 \mathrm{~dB} A R$ beamwidth of $136^{\circ}$ for every phi cut, wider than current state-of-the-art [1]. Moreover, the proposed antenna array presents a $50 \Omega$ impedance with no impedance matching network, and requires only two phase shifters to steer, significantly reducing the design complexity. The antenna's $10 \mathrm{~dB}$ return loss bandwidth is from $843 \mathrm{MHz}$ to $880 \mathrm{MHz}$, covering the lower band UHF RFID frequencies in the European Telecommunications Standards Institute (ETSI) region.

Index Terms-Antenna arrays, Phased arrays, Radiofrequency identification, UHF antennas, UHF propagation
\end{abstract}

\section{INTRODUCTION}

Ultra-high-frequency (UHF) RFID has been widely adopted in inventory checking, access control and indoor localization [2], [3]. In a UHF RFID system, one of the most crucial components is the reader antenna, its properties directly affect the overall performance of the system. As UHF RFID systems have become increasingly popular in indoor applications, signal nulls caused by multi-path fading have become the key problems that need to be overcome: when signals are reflected from walls or other objects commonly found indoors, they might interfere with the direct line-of-sight signal. When these interfering signals are $180^{\circ}$ out of phase with the LoS signal, they would add destructively, resulting in a power that is potentially below the RFID tag sensitivity.

Significant research efforts have been made to overcome this problem, of which beam-scanning antennas have proven to be an effective approach, providing better signal coverage and higher read rates [4]-[10]. Steering antennas are also useful for location tracking and cause less interference to adjacent systems allowing operation at a higher effective isotropic radiated power (EIRP) compared to omni-direction antennas under the Federal Communications Commission (FCC) and ETSI regulations. However, many of the previously proposed antennas are linearly polarized ( [5], [8]) and [6]-[9] only allow one dimensional (1D) beam steering. Their $3 \mathrm{~dB}$ AR beamwidth are narrow compared to this work as in Table I. On the contrary, an antenna array capable of 2D beam steering is proposed in this work: the proposed antenna is able to steer its beam from $0^{\circ}$ to $360^{\circ}$ in the azimuth, and $-40^{\circ}$ to $40^{\circ}$ in the elevation. This added dimension of freedom could allow the antenna to provide a wide area of coverage when mounted on a ceiling facing down to an interrogation zone below.

An important parameter for RFID reader antennas is the axial ratio (AR). For most applications, circularly polarized (CP) antennas are preferred as in many practical applications, the tag antennas are linearly polarized (LP) and can be arbitrarily oriented. Therefore, the use of a circularly polarized reader antenna with a good AR maximises the likelihood to tag detection. The proposed antenna has a very low (close to 0 dB) AR around its beam maxima, which follows the peak gain during beam steering. The antenna's AR doesn't deteriorate when the beam is tilted with a large $\theta$ angle, giving the antenna a wide equivalent $3 \mathrm{~dB}$ AR beamwidth of $136^{\circ}$, in every $\phi$ cut.

Table I gives a comparison of this work to antennas proposed in literature:

TABLE I

A COMPARISON OF THE PROPOSED ANTENNA WITH ANTENNAS IN LITERATURE

\begin{tabular}{lccccl}
\hline & {$[7]$} & {$[8]$} & {$[6]$} & {$[10]$} & This Antenna \\
\hline Beam-scanning & 1D & $1 \mathrm{D}$ & $1 \mathrm{D}$ & $2 \mathrm{D}$ & $2 \mathrm{D}$ \\
3dB AR beamwidth & $25^{\circ}$ & LP & $60^{\circ}$ & $38^{\circ}$ & $136^{\circ}$ \\
Needs matching & Yes & Yes & No & Yes & No \\
Network & 7.1 & 6.5 & 10 & 12.5 & 10.5 \\
Gain (dB) & & & &
\end{tabular}

The rest of this paper is organized as follows: Section II explains the antenna design, including parameter selection, design and optimization procedures and full-wave simulation. Section III presents the measurement results of the manufactured antenna and compares them with simulation results and those of a conventional 2-by-2 phased array. A conclusion is given in section IV.

\section{Antenna Design And Performance}

It was first proposed in [11] that circular polarization can be stimulated by adding a branch wire with a certain length at the correct location to a loop antenna. While the detailed analysis can be found in [11], [12], the conclusion is that loop antennas become circularly polarized provided that a travelingwave-type current distribution is created on the antenna. This means that the (maximum) current amplitude should be the same on different segments of the loop antenna while phase should be linear.

A physical interpretation can be achieved by considering the current distributions: For a conventional loop antenna with 
a circumference of one wavelength and a sinusoidal source as shown in Fig. 1, a standing wave is formed on the loop with two peaks located on the $\mathrm{Y}$ axis (the feed point is on the $\mathrm{X}$ axis). This can be considered to be analogues to a bent dipole. Using vector potential analysis or full-wave simulation, it can be shown that the predominant electric component would be $E_{y}$. On the contrary, for a loop antenna with traveling-wave-type current distribution as shown in Fig. 2 and 3, because the current amplitude is the same on different segments of the antenna while the phase is linear, the current peaks travel around the antenna. This can be seen as analogues to physically rotating a conventional loop antenna around the $\mathrm{Z}$ axis, along with the predominant electric field components. As a result, the antenna becomes circularly polarized.

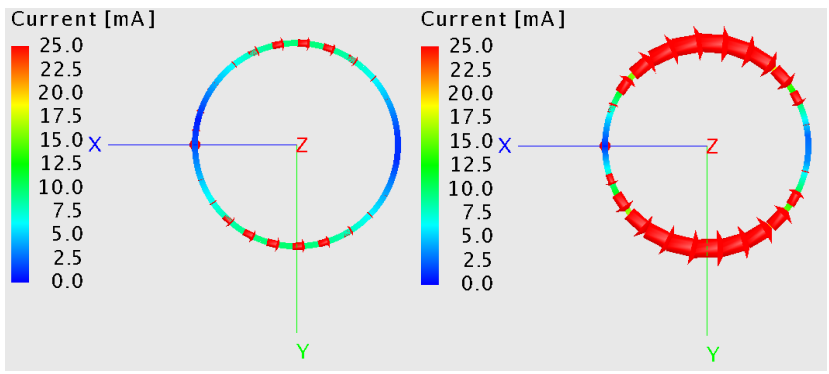

Fig. 1. Current distribution for a conventional $1 \lambda$ loop antenna with standingwave-type current. Left: $t=0$, right: $t=T / 4$

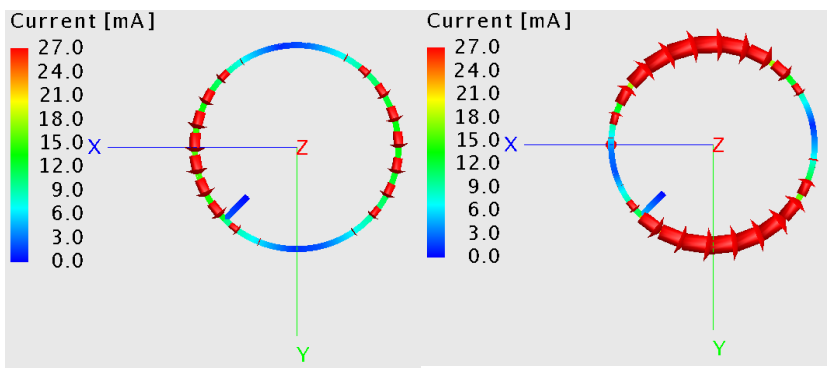

Fig. 2. Current distribution for a $1 \lambda$ loop antenna with traveling-wave-type current. Left: $\mathrm{t}=0$, right: $\mathrm{t}=\mathrm{T} / 4$

As analyzed in [13] a $1 \lambda$ loop antenna generates a fixed axial beam pattern with its peak located at $\theta=0$. To form an antenna with a steerable beam, two extra rings with perimeters of approximately $2 \lambda$ and $3 \lambda$ are added in the design. When operating alone, each of these two rings produces a conical beam. By manipulating the relative phase and amplitude of these three rings, constructive and destructive interference can generate a single beam who's direction can be steered.

\section{A. Antenna Design}

The proposed antenna is shown in Fig. 4, which is made of three layers: The radiating layer is formed by three concentric copper rings. Their perimeters are around $1 \lambda, 2 \lambda$ and $3 \lambda$ respectively, and can be fine-tuned to improve the AR performance. Here $\lambda$ is the wavelength of the central operating frequency in free space $(866 \mathrm{MHz}$ in this design). Each of
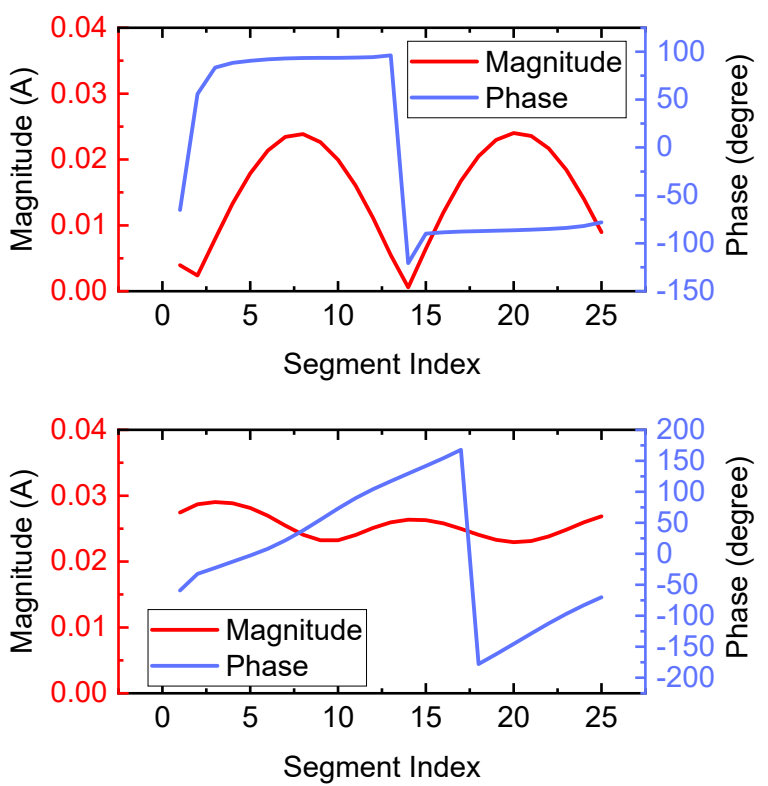

Fig. 3. Amplitude and phase distribution of a $1 \lambda$ loop antenna. Top: a conventional loop with standing-wave current distribution, bottom: a loop with perturbation element showing traveling-wave current distribution

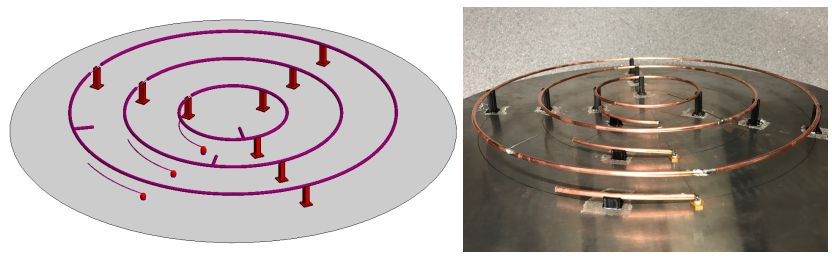

Fig. 4. The proposed antenna structure. Left: The antenna modelled in FEKO. Right: The manufactured antenna

these metal rings has a perturbation line (PL), the length of which are $0.065 \lambda, 0.057 \lambda$ and $0.061 \lambda$ respectively. These perturbation lines are placed at $\varphi=45^{\circ}, 22.5^{\circ}$ and $-45^{\circ}$ respectively, to achieve the best AR performance. To support these metal rings, some Polylactic Acid (PLA) pillars with a height of $0.063 \lambda$ are manufactured through $3 \mathrm{D}$ printing.

Below the radiating layer is the feeding layer. The feed of the antenna is through electromagnetic coupling: three elliptic copper arcs are placed directly under three circles without touching them. These copper arcs are supported by

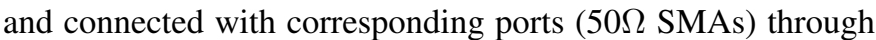
the ground plane and their curvatures are the same as the corresponding circles above. The length and height of these arcs are approximately $0.23 \lambda$ and $0.02 \lambda$ respectively, and can be adjusted for impedance matching purposes.

Below the feeding layer is the ground plane, which is a round aluminum sheet with $45 \mathrm{~cm}$ diameter and $1 \mathrm{~mm}$ thickness. It can be proven using numerical analysis or full-wave simulation that these design parameters work for different central frequencies [6], [11], [12]. 


\section{B. Design Procedures}

When it comes to the design procedures of the antenna, although some analysis has been given in [6], [11], [12] about the condition to stimulate circular polarization, in practice, the exact values of antenna parameters have to be decided through optimization. Take the inner ring as an example: an isolated inner ring is created with initial parameters (its perimeter is set to be $0.988 \lambda$ while the perturbation line length is set to be $0.05 \lambda$ ). The first step is to find the correct $\varphi$ angle to place the perturbation line. This is done by using the parameter sweep function of a full-wave simulation software (FEKO [14]) to choose the right $\varphi$ angle where the axial ratio of the generated beam becomes minimal, as shown in Fig. 5. From [13], it is known that a loop antenna with $1 \lambda$ perimeter generates an axial beam. Thus to optimize the axial ratio at its peak position, the observation is set at $(\theta, \varphi)=(0,0)$ in Fig. 5 . Alternatively, as it is explained in the above section, adding a perturbation element can be equally seen as physically rotating the conventional loop antenna around the $\mathrm{Z}$ axis, it can be expected that the resulting radiation pattern is also a rotation of the original pattern. As a result, to find the right observation point for axial ratio optimization, one can do a simulation using a loop antenna with the same perimeter but without a perturbation line, and use its peak location as the observation point.
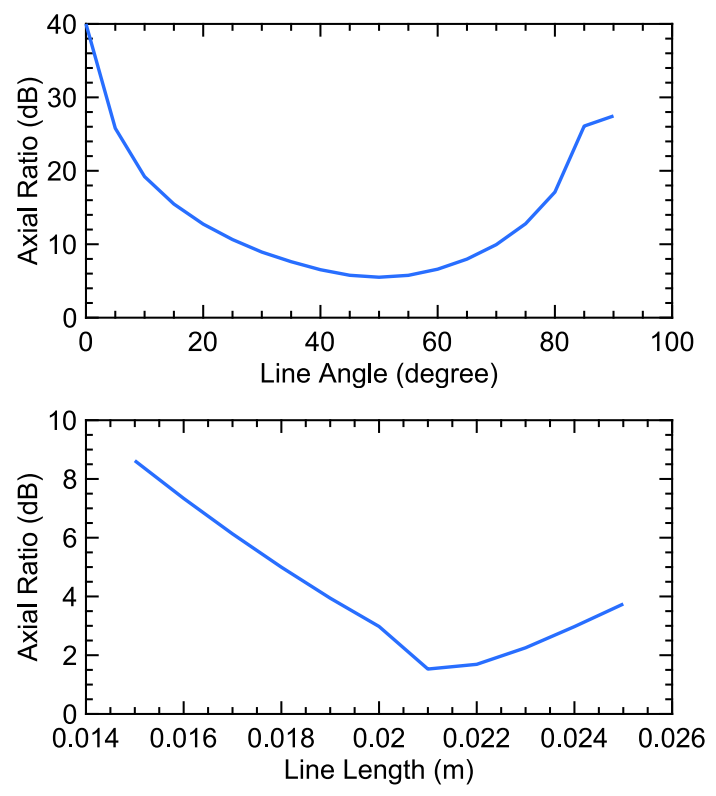

Fig. 5. Axial ratio of the inner ring (the observation point is $(\theta, \varphi)=(0,0)$ ). Top: AR versus the $\varphi$ angle of the perturbation line, with loop perimeter fixed at $0.988 \lambda$ and perturbation line length fixed at $0.05 \lambda$, bottom: AR versus the perturbation line length, with loop perimeter fixed at $0.988 \lambda$ and line angle fixed at $\varphi=45^{\circ}$. These parameters needs to be fine-tuned after adding all 3 rings.

After the $\varphi$ angle has been chosen, similar procedures are used to determine the optimal length for the perturbation line, the result of which is shown in Fig. 5. The length and height of the antenna's feed line would affect the impedance match performance, and can be optimized by a similar method. The above procedures are repeated for the second and the third ring. One advantage of the proposed antenna is, these three metal rings can be designed and optimized separately. After installing them together, neither their impedance match nor AR performance would change significantly and only some minor adjustments are needed to optimize the antenna as a whole.

A list of final parameters is given in table II

TABLE II

ANTENNA PARAMETERS

\begin{tabular}{llll}
\hline & First Ring & Second Ring & Third Ring \\
\hline Perimeter & $0.988 \lambda$ & $1.984 \lambda$ & $2.984 \lambda$ \\
PL Angle & $45^{\circ}$ & $22.5^{\circ}$ & $-45^{\circ}$ \\
PL Length & $0.058 \lambda$ & $0.065 \lambda$ & $0.061 \lambda$ \\
Feed line Length & $0.23 \lambda$ & $0.23 \lambda$ & $0.24 \lambda$ \\
Feed line Height & $0.022 \lambda$ & $0.017 \lambda$ & $0.018 \lambda$ \\
\hline
\end{tabular}

\section{Beam-Scan Configurations}

Using calculations in [12], it is revealed that a tilted beam is formed along the $\varphi=90^{\circ}$ plane with its $10.9 \mathrm{~dB}$ peak gain located at $\theta=30^{\circ}$, which agrees with full-wave simulation results. If the input phase of the middle ring is kept constant while the phase of the signal fed to the inner ring is shifted by $+\Delta \phi$ with respect to it and by $-\Delta \phi$ for the outer ring, the resulting beam would rotate within the azimuth plane (anticlockwise) by $\Delta \phi$ degree as shown in figure 6 .

Beam-steering in the zenith plane is achieved by changing the amplitude ratio fed to three rings while maintaining the same total input power. A tilting angle of $0^{\circ}$ is achieved when an amplitude ratio of 9:1 (inner to middle and outer) is applied. Whereas a ratio of $0: 10$ produces a $40^{\circ}$ tilting angle.

By continuously tuning the amplitude ratio, the tilting angle can be seamlessly adjusted between $0^{\circ}$ and $40^{\circ}$, as shown in figure 7 . The gain of the antenna remains relatively stable during this tilting process, reaching a maximum of $10.9 \mathrm{~dB}$ at $\theta=30^{\circ}$, and a minimum value of $9.24 \mathrm{~dB}$ at $\theta=40^{\circ}$.

The minimum $\mathrm{AR}$ of the antenna is close to $0 \mathrm{~dB}$ and is always around the antenna's steerable beam. Simulation reveals that the $3 \mathrm{~dB}$ AR beamwidth of the antenna is $68^{\circ}$, when its peak gain is at $\theta=40^{\circ}$. Since the antenna's beam can be steerable between $\pm 40^{\circ}$ in $\theta$, an equivalent $3 \mathrm{~dB}$ AR beamwidth of $136^{\circ}$ is achievable in every phi cut.

\section{Simulation AND MEAsurement}

The designed antenna is manufactured and characterized using MVG StarLab ${ }^{\circledast}$ antenna chamber [15] and a custom PCB board based on Mini-Circuits ${ }^{\circledast}$ SPHSA-152+ phase shifters [16] and qorvo ${ }^{\circledast}$ RFSA3713 digital attenuators [17]. The results are compared with simulation and are shown in Fig. 6 to Fig. 8. It is clear that the validity of the proposed antenna is verified: the measured curves match well with simulation results, indicating that the antenna's beam can be steered from 0 to 360 degree in the azimuth plane, and -40 to 40 degree in 
the zenith plane, as predicted by theory. In Fig. 8, it is clear that during the beam steering process, the AR beam follows the beam direction of the gain, creating a $3 \mathrm{~dB}$ AR beamwidth of around $136^{\circ}\left( \pm 68^{\circ}\right)$

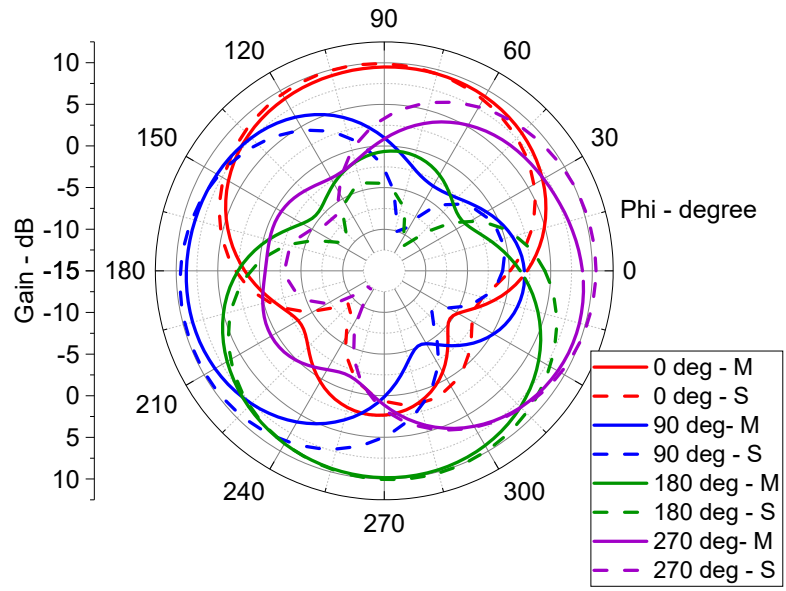

Fig. 6. Simulated and measured total gain of the proposed antennad against $\varphi$ at $\theta=30^{\circ}$ cut, with different values of phase difference. In the graph, a suffix of $\mathrm{M}$ means measured result while $\mathrm{S}$ means simulated

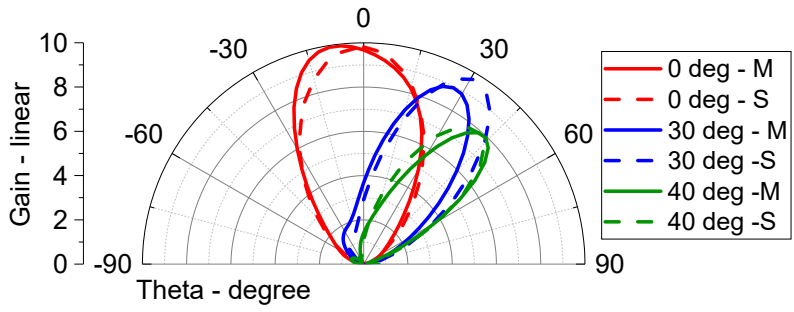

Fig. 7. Simulated and measured total gain of the proposed antenna against $\theta$ at $\varphi=90^{\circ}$ cut, with different values of the voltage ratio. In the graph, a suffix of $\mathrm{M}$ means measured result while $\mathrm{S}$ means simulated

The proposed antenna is compared with a commonly available, conventional $2 \times 2$ phased array antenna as shown in Fig. 9. The measurement results of this antenna are shown in Fig. 11 to Fig. 13.

The proposed antenna has several key advantages over the commercial antenna:

- The proposed antenna has a higher gain (approximately $10 \mathrm{dBic}$ ) than the conventional antenna (approximately $6 \mathrm{dBic})$, which is likely to be due to the air dielectric used in the circular array, instead of lossy ceramic in the patches. Moreover, the gain and radiation pattern of the proposed antenna stays relatively stable $(9.4 \mathrm{dBic}$ to $10.5 \mathrm{dBic}$ ) during beam-steering process whereas there are notable changes to those of the commercial antenna, as shown in Fig. 11.

- The proposed antenna achieved a $3 \mathrm{~dB}$ axial ratio of $136^{\circ}$, whereas the commercial antenna has an average AR of 4 $\mathrm{dB}$ within its beamwidth, except for the situation when the beam is tilted to $\theta=30^{\circ}$.

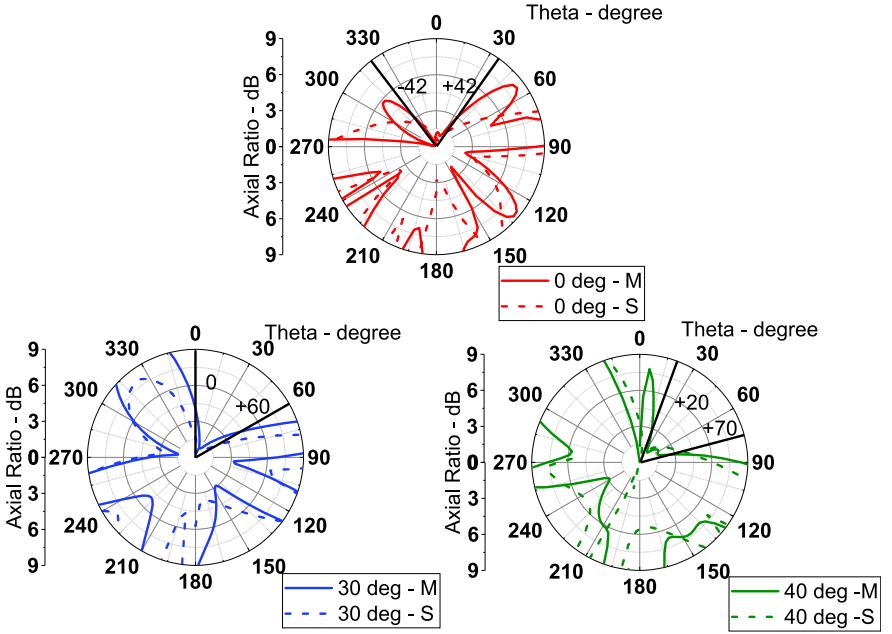

Fig. 8. Simulated and measured axial ratio of the proposed antenna against $\theta$ at $\varphi=90^{\circ}$ cut, with the peak gain direction at $\theta=0^{\circ}, 30^{\circ}$ and $40^{\circ}$ respectively. In the graph, a suffix of $\mathrm{M}$ means measured result while $\mathrm{S}$ means simulated

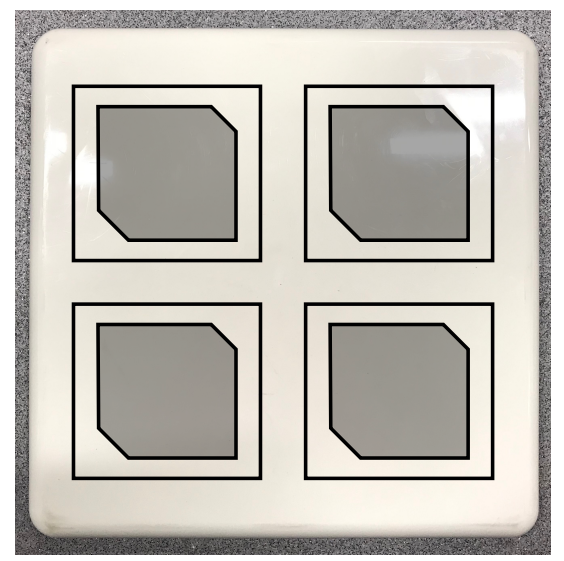

Fig. 9. A conventional $2 \times 2$ phased array antenna made of 4 off-the-shelf array elements. Hidden array elements are indicated

- The proposed antenna requires no matching network and only 2 rather than 3 phase shifters to operate, which greatly simplifies the design.

TABLE III

A COMPARISON OF THE PROPOSED ANTENNA WITH A COMMERCIAL ANTENNA

\begin{tabular}{lll}
\hline & A commercial antenna & The proposed antenna \\
\hline Size & $30 \mathrm{~cm} \times 30 \mathrm{~cm}$ & $45 \mathrm{~cm}$ (diameter) \\
Gain & $6 \mathrm{dBic}$ & $10.5 \mathrm{dBic}$ \\
$3 \mathrm{~dB}$ AR beamwidth & $45^{\circ}$ (maximum) & $136^{\circ}$ \\
Needs Matching & Yes & No \\
Network & & 2 \\
$\begin{array}{l}\text { Number of Phase } \\
\text { Shifters }\end{array}$ & 3 & \\
\hline
\end{tabular}

\section{CONCLUSION}

A novel antenna with $2 \mathrm{D}$ beam-scan ability, wide axial ratio beamwidth and simple configuration is designed, simulated 
and measured. It is compared with both beam-scan antennas in literature and a commercial phased array antenna, and is shown to possess key advantages in terms of design complexity, beam-scan abilities and AR performance. With an ultra-wide $3 \mathrm{~dB}$ AR beamwidth of $136^{\circ}, 2 \mathrm{D}$ beam-scan from $0^{\circ}$ to $360^{\circ}$ in the azimuth plane and $0^{\circ}$ to $40^{\circ}$ in the elevation plane, the proposed antenna could be used in UHF RFID systems to provide better signal coverage. Moreover, as the antenna's radiation pattern is predictable and stable during the beamscanning process, it is suitable to be used in RFID localization schemes where the beam-steering ability is involved [18], [19].

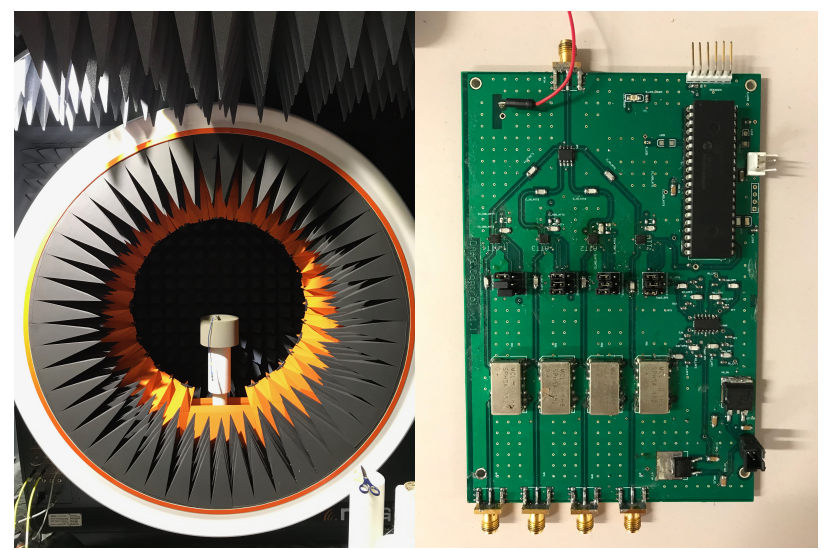

Fig. 10. Left: MVG StarLab ${ }^{\circledR}$ antenna characterization chamber, right: a custom PCB board with Mini-Circuits ${ }^{\circledR}$ SPHSA-152+ phase shifters [16] and qorvo ${ }^{\circledR}$ RFSA3713 digital attenuators [17]. Only two phase shifters are used for the proposed antenna.

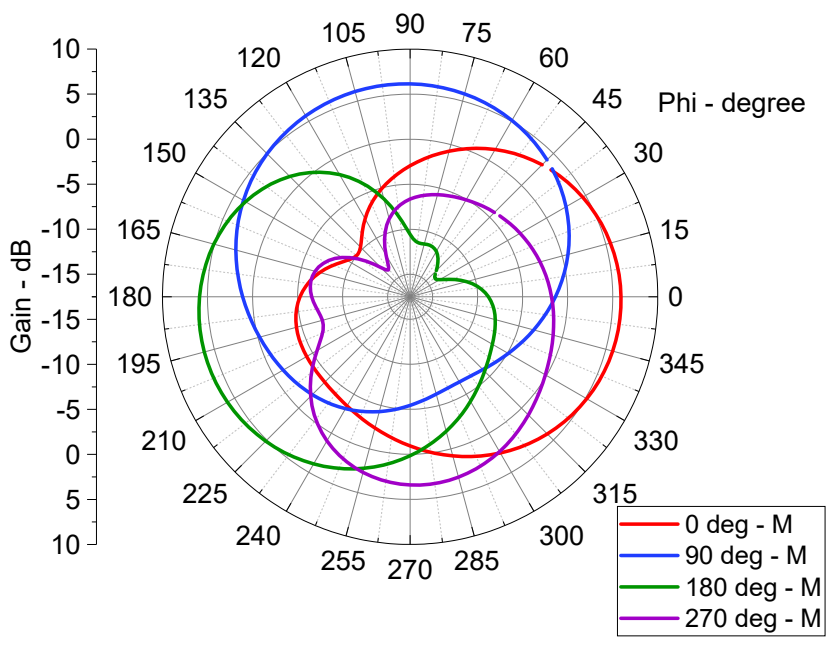

Fig. 11. Measured total gain of the commercial antenna against $\varphi$ at $\theta=30^{\circ}$ cut, with different values of phase difference. In the graph, a suffix of $\mathrm{M}$ means measured result while $\mathrm{S}$ means simulated

\section{REFERENCES}

[1] P. Wang, G. Wen, J. Li, Y. Huang, L. Yang, and Q. Zhang, "Wideband circularly polarized uhf rfid reader antenna with high gain and wide axial ratio beamwidths," Progress In Electromagnetics Research, vol. 129, pp. 365-385, 2012.

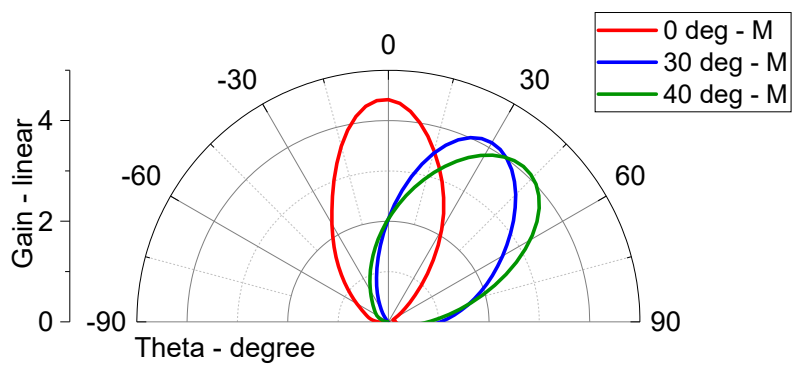

Fig. 12. Measured total gain of the commercial antenna against $\theta$ at $\varphi=90^{\circ}$ cut, with different values of the voltage ratio. In the graph, a suffix of M means measured result while $\mathrm{S}$ means simulated

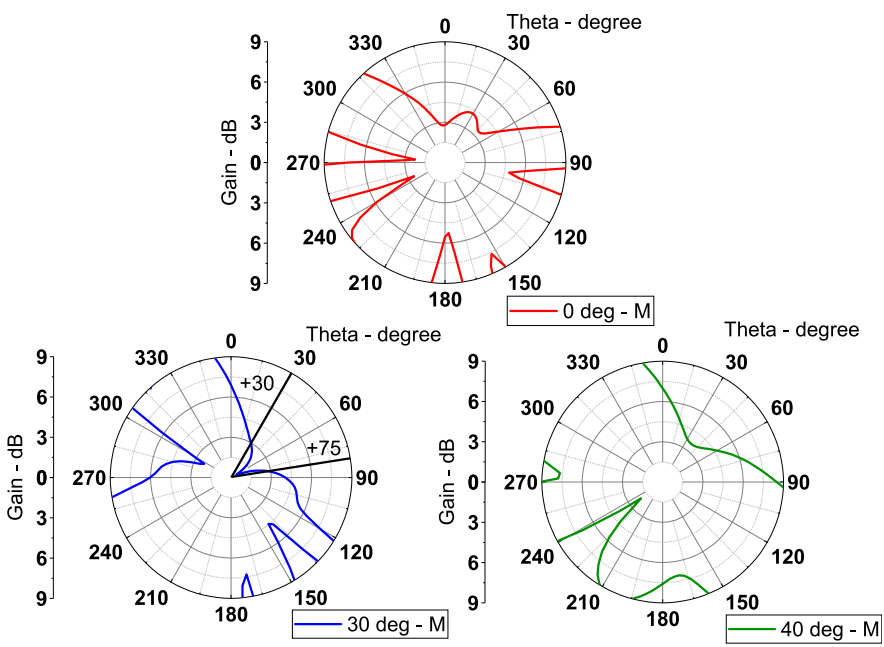

Fig. 13. Measured axial ratio of the commercial antenna against $\theta$ at $\varphi=90^{\circ}$ cut, with the peak gain direction at $\theta=0^{\circ}, 30^{\circ}$ and $40^{\circ}$ respectively. In the graph, a suffix of $\mathrm{M}$ means measured result while $\mathrm{S}$ means simulated

[2] A. P. Sample, D. J. Yeager, P. S. Powledge, A. V. Mamishev, and J. R. Smith, "Design of an rfid-based battery-free programmable sensing platform," IEEE transactions on instrumentation and measurement, vol. 57, no. 11, pp. 2608-2615, 2008.

[3] S. Siachalou, A. Bletsas, J. Sahalos, and A. G. Dimitriou, "Rssi-based maximum likelihood localization of passive rfid tags using a mobile cart," in 2016 IEEE Wireless Power Transfer Conference (WPTC). IEEE, 2016, pp. 1-4.

[4] W.-S. Lee, S.-T. Khang, W.-S. Lee, and J.-W. Yu, "Hemispheric coverage multi-beam switched antenna array using a 4-port feeding network for uhf rfid dead zone avoidance," in 2013 Asia-Pacific Microwave Conference Proceedings (APMC). IEEE, 2013, pp. 254-257.

[5] X. Li, H. Zhu, Q. Li, and W. Feng, "Beam scanning uhf rfid reader antenna design for metal environment application," in 2018 IEEE International Conference on Service Operations and Logistics, and Informatics (SOLI). IEEE, 2018, pp. 108-111.

[6] K. Hirose, S. Okazaki, and H. Nakano, "Double-loop antennas for a circularly polarized tilted beam," Electronics and Communications in Japan (Part I: Communications), vol. 86, no. 12, pp. 12-20. [Online]. Available: https://onlinelibrary.wiley.com/doi/abs/10.1002/ecja.10132

[7] C. Liu, S. Xiao, Y.-X. Guo, Y.-Y. Bai, and B.-Z. Wang, "Broadband circularly polarized beam-steering antenna array," IEEE Transactions on Antennas and Propagation, vol. 61, no. 3, pp. 1475-1479, 2012.

[8] S.-L. Chen, P.-Y. Qin, W. Lin, and Y. J. Guo, "Pattern-reconfigurable antenna with five switchable beams in elevation plane," IEEE Antennas and Wireless Propagation Letters, vol. 17, no. 3, pp. 454-457, 2018.

[9] M. Abbak and I. Tekin, "Microstrip patch antenna array for range extension of rfid applications," in 2008 IEEE Antennas and Propagation 
Society International Symposium. IEEE, 2008, pp. 1-4.

[10] W. Hu, G. Wen, D. Inserra, Y. Huang, J. Li, and Z. D. Chen, "A circularly polarized antenna array with gain enhancement for long-range uhf rfid systems," Electronics, vol. 8, no. 4, p. 400, 2019.

[11] T. Nakamura and S. Yokokawa, "Loop antenna with a branch wire for circular polarization," Electronics and Communications in Japan (Part I: Communications), vol. 70, no. 11, pp. 110-117, 1987.

[12] H. Nakano, "A numerical approach to line antennas printed on dielectric materials," Computer physics communications, vol. 68, no. 1-3, pp. 441450, 1991.

[13] C. A. Balanis, Antenna theory: analysis and design. John wiley \& sons, 2016.

[14] E. S. . S.-S. P. Ltd. (2014, may) FEKO user manual. Accessed on: May 5, 2019. [Online]. Available: http://altairuniversity.com/wpcontent/uploads/2015/03/UserManual.pdf

[15] MVG. (2019) StarLab. Accessed on: May 5, 2019. [Online]. Available: https://www.mvg-world.com/en/products/field_product_family/antennameasurement-2/starlab

[16] Mini-Circuits. (2019, may) Surface mount phase shifter sphsa152+. Accessed on: May 5, 2019. [Online]. Available: https://ww2.minicircuits.com/pdfs/SPHSA-152+.pdf

[17] qorvo. (2019, may) 5 - $6000 \mathrm{mhz}$ serial controlled digital step attenuator, 7-bit, $0.25 \mathrm{db}$ steps. Accessed on: May 5, 2019. [Online]. Available: https://www.qorvo.com/products/p/RFSA3713

[18] J. Vongkulbhisal and Yan Zhao, "An rfid-based indoor localization system using antenna beam scanning," in 2012 9th International Conference on Electrical Engineering/Electronics, Computer, Telecommunications and Information Technology, May 2012, pp. 1-4.

[19] R. Kronberger, T. Knie, R. Leonardi, U. Dettmar, M. Cremer, and S. Azzouzi, "Uhf rfid localization system based on a phased array antenna," in 2011 IEEE International Symposium on Antennas and Propagation (APSURSI), July 2011, pp. 525-528. 